\title{
Gold Nanoparticle/Tetrapyridylporphyrin Hybrid Material: Spectroscopic and Electrocatalytic Properties and Sensor Application
}

\author{
Jonnatan J. Santos, ${ }^{\oplus *, a}$ Sergio H. Toma, ${ }^{\circledR a}$ Rebeca E. Yatsuzuka ${ }^{\oplus a}$ and Koiti Araki ${ }^{\oplus a}$ \\ ${ }^{a}$ Departamento de Química Fundamental, Instituto de Química, Universidade de São Paulo, \\ Av. Lineu Prestes, 748, Butantã, 05508-000 São Paulo-SP, Brazil
}

\begin{abstract}
An electrocatalytic active hybrid material prepared by successive stepwise coordinative deposition of $20 \mathrm{~nm}$ diameter gold nanoparticles (AuNP) with 5,10,15,20-tetra(4-pyridyl)$21 \mathrm{H}, 23 \mathrm{H}$-porphine (TPyP) on fluorine-doped tin oxide (FTO) substrates previously modified with 3-mercaptopropylsilane is described. Their interaction and assembly were studied by UV-Vis spectroscopy and surface enhanced Raman spectroscopy to understand the kinetics as well as the conformation on the porphyrin macrocycle on the electrode surface as a function of its concentration. The hybrid nanomaterial was a build-up in reproducible successive deposition steps as confirmed by the linear rise of the absorption bands assigned to AuNP and TPyP as a function of the number of AuNP/porphyrin hybrid deposition steps, as supported by scanning electron microscopy (SEM) images. Electrodes modified with the hybrid nanomaterials were prepared and characterized by cyclic voltammetry (CV) and electrochemical impedance spectroscopy (EIS), as well as their electrocatalytic properties for the oxidation of nitrite and sulfite investigated. FTO electrodes modified with the AuNP/TPyP hybrid showed remarkable electrocatalytic activity as confirmed by reproducible and sensitive amperometric responses for sensing nitrite and sulfite in comparison to the bare electrode while reducing overpotentials respectively to 0.8 and $0.4 \mathrm{~V}$ and improving the dynamic range (linear response allowing quantification in the 50 to $500 \mu \mathrm{mol} \mathrm{\textrm {L } ^ { - 1 }}$ range) for detection of analytes.
\end{abstract}

Keywords: gold nanoparticle, porphyrin, SERS, LSPR, coordinative assembly, modified electrode, electrocatalysis

\section{Introduction}

Gold nanoparticles are especially known for their optoelectronic properties and implications such as localized surface plasmon resonance (LSPR), surfaceenhanced Raman scattering (SERS), and surface-enhanced fluorescence (SEF) among other effects. ${ }^{1-4}$

Although the phenomena related to plasmons are of interest for photon-based spectroscopy measurements, the intrinsic characteristics of gold, such as high conductivity, low reactivity and the high surface area of nanoparticles make them very interesting for use in electrochemical and electroanalytical measurements. ${ }^{5}$

The use of nanoparticles in electrochemistry depends on their deposition on an electrode with the consequent formation of films. Among the many ways to prepare and

*e-mail: jonnatan@iq.usp.br

Dedicated to Prof Henrique Eisi Toma on the occasion of his $70^{\text {th }}$ birthday. deposit hybrids of nanoparticles on a surface, two strategies stand out: in situ or ex situ.

In the in situ strategy, gold nanoparticles are prepared/ deposited directly on the electrode, reducing a gold salt solution, electrochemically, in the presence of organic or inorganic species. ${ }^{67}$ In contrast, through the ex situ strategy, nanoparticles are previously generated by chemical methodologies (reduction of gold salts using citrate, ascorbic acid, borohydride, etc. $)^{8}$ and later deposited by self-assembly strategies with the molecular linker.,

While the in situ strategy has speed and simplicity as its main advantage, the ex situ strategy allows the control of the particle size, the functionalization and deposition process and, consequently, the properties of the film. Thus, the ex situ strategy is the most interesting from the point of view of nanotechnology.

In fact, the ex situ strategy, based on the simple interaction of van der Waals, coordinative or electrostatic interactions, can be used to make the deposition, but the most convenient is to use bridge molecules capable of 
electronically and physically connecting the nanoparticles, stabilizing the film and ensuring higher conductivity and control of interparticle distances. ${ }^{11}$

Among the possible bridge molecules with redox activity are porphyrins, important macrocycles present in biological systems, involved in the transport and storage of electrons and oxygen, in addition to important redox processes, such as respiration and photosynthesis. ${ }^{12}$ The 5,10,15,20-tetra (4-pyridyl)-21H,23H-porphine, for example, also known as tetrapyridylporphyrin (TPyP), has interesting photochemical, catalytic and electrocatalytic properties that can be enhanced by the bonding of transition metal complexes to four pyridyl substituting peripherals. ${ }^{13,14}$ In this way, several electrode modification strategies have been carried out to take advantage of the properties of porphyrins and their supramolecular systems for the generation of electroactive materials that can be used, for example, in the detection of nitrite found in meat, sulfite in wines, ascorbic acid in juices, among others. ${ }^{15,16}$ In addition, hybrid systems with porphyrins have been widely used in photocatalysis. ${ }^{17,18}$

In this study, we demonstrate how materials with such different characteristics (a low polarity organic molecule and aqueous dispersed nanoparticles) can interact, and how this interaction is dependent on relative porphyrin/ nanoparticle concentration. This interaction was used to form molecular nanostructured hybrid materials that can profoundly modify the electrode properties and, finally, how they can be utilized to electrocatalytic sensing of relevant analytes, such as nitrite and sulfite.

\section{Experimental}

\section{Materials}

Deionized water (resistivity $=18.2 \mathrm{M} \Omega \mathrm{cm}$ ) was obtained using a MilliUni deionizer, model Direct-Q 3U. Nitric acid ( $\geq 65$ wt. $\%$ ), hydrochloric acid ( $\geq 37$ wt.\%), potassium chloride $(\mathrm{KCl}, 99 \%)$, hydrogen peroxide $(30 \% \mathrm{~m} / \mathrm{m})$, ammonium hydroxide $(28-30 \%)$ tetrachloroauric acid trihydrate (52 wt.\% of Au), trisodium citrate hydrate ( $\geq 98 \%), 5,10,15,20$-tetra(4-pyridyl)-21H,23H-porphine (TPyP, 97\%), (3-mercaptopropyl)trimethoxysilane (MPTS, 95\%), potassium hexacyanoferrate(II) trihydrate $\left(\mathrm{K}_{4}\left[\mathrm{Fe}(\mathrm{CN})_{6}\right] \cdot 3 \mathrm{H}_{2} \mathrm{O}, 98.5 \%\right)$, potassium hexacyanoferrate(III) $\left(\mathrm{K}_{3}\left[\mathrm{Fe}(\mathrm{CN})_{6}\right], 99 \%\right)$ were all obtained from Sigma-Aldrich (São Paulo, Brazil) and used as received.

\section{Equipment}

The UV-Vis spectra were obtained in a Hewlett Packard model 8453A (United States of America) spectrophotometer using $10.0 \mathrm{~mm}$ optical path quartz cells or, directly, from the AuNP/TPyP hybrid material deposited on fluorine-doped tin oxide (FTO) electrodes, a bare FTO electrode was used for blank spectrum.

The SERS spectra were acquired using a Raman spectrometer from InPhotonics, model InPhotote, (United States of America) equipped with a $785 \mathrm{~nm}$ laser (laser spot diameter $=100 \mu \mathrm{m}$, area $=7850 \mu \mathrm{m}^{2}$ ), using an accumulation time of $120 \mathrm{~s}$ and back-scattering configuration.

Scanning electron microscopy (SEM) images were registered using a field emission gun scanning electron microscope (FEG-SEM) from JEOL, model-JSM-7401F (Japan), at a typical acceleration voltage of $3 \mathrm{kV}$. The images were registered directly from AuNP/TPyP hybrid modified FTO electrodes.

Electrochemical measurements were performed using a potentiostat/galvanostat AutoLab PGSTAT30 (EcoChemie, The Netherlands) and a conventional three electrodes system.

Electrochemical impedance spectroscopy (EIS) measurements were performed in aqueous solution containing $0.5 \mathrm{~mol} \mathrm{~L}^{-1} \mathrm{KNO}_{3}, 1 \times 10^{-3} \mathrm{~mol} \mathrm{~L}^{-1}$ of $\mathrm{K}_{3}\left[\mathrm{Fe}(\mathrm{CN})_{6}\right]$ and $\mathrm{K}_{4}\left[\mathrm{Fe}(\mathrm{CN})_{6}\right]$, at the formal potential of the $\left[\mathrm{Fe}^{\mathrm{III} / \mathrm{II}}(\mathrm{CN})_{6}\right]$ redox couple $(0.36 \mathrm{~V}$ vs standard hydrogen electrode (SHE)), setting the AC amplitude to $20 \mathrm{mV}$ and scanning the frequency in the $0.05 \mathrm{~Hz}$ to $10.0 \mathrm{kHz}$ range, as previously reported. ${ }^{19}$ The impedance spectra were fitted using the FRA software (AUTOLAB, Eco Chemie, The Netherlands).

Cyclic voltammetry (CV) and electrochemical impedance spectroscopy experiments were carried out in aqueous solution using a conventional threeelectrodes configuration. This system consisting of an $\mathrm{Ag} / \mathrm{AgCl}\left(1.0 \mathrm{~mol} \mathrm{~L}^{-1} \mathrm{KCl}\right)$ as reference electrode $\left(\mathrm{E}_{0}=0.222 \mathrm{~V} v s\right.$. SHE), a coiled platinum wire auxiliary electrode and a FTO electrode (unmodified or modified by AuNPs and TPyP) as working electrode with geometrical area of $0.25 \mathrm{~cm}^{2}$, and $\mathrm{KNO}_{3}\left(0.1 \mathrm{~mol} \mathrm{~L}^{-1}\right)$ as supporting electrolyte.

\section{Synthesis of citrate gold nanoparticles (AuNP)}

Gold nanoparticles (AuNP) were obtained by the Turkevitch/Frens method ${ }^{20,21}$ by heating $100 \mathrm{~mL}$ of a $0.02 \%$ $(\mathrm{m} / \mathrm{v}) \mathrm{HAuCl}_{4}$ solution to boiling, under vigorous stirring. Then, $2.75 \mathrm{~mL}$ of a $1 \%(\mathrm{~m} / \mathrm{v})$ trisodium citrate solution was added at once.

Immediately, the solution turned dark blue to purple and, after approximately 1 min under heating and stirring, became red-colored. UV-Vis spectroscopy analysis indicated the presence of a maximum plasmon resonance 
band at $521 \mathrm{~nm}$, corresponding to a dispersion of about $26 \mathrm{~nm}$ diameter AuNPs.

Kinetic monitoring of AuNP/TPyP interaction by UV-Vis spectroscopy

The kinetic reaction between AuNPs and TPyP was carried out using a $10.0 \mathrm{~mm}$ quartz cuvette containing AuNP suspension $(3.0 \mathrm{~mL})$ and by adding different volumes of TPyP solution (concentration equal to $1 \times 10^{-3} \mathrm{~mol} \mathrm{~L}^{-1}$ or $1 \times 10^{-4} \mathrm{~mol} \mathrm{~L}^{-1}$ in dichloromethane) whilst stirring. The evolution of the reaction was monitored spectrophotometrically at $1 \mathrm{~s}$ intervals by using the HP-8453A (United States of America) diode array spectrophotometer in the kinetic mode.

\section{Modification of FTO electrodes with AuNP/TPyP hybrid material}

The FTO conductive glass electrodes were modified with the nanostructured material prepared in-situ by stepwise coordinative assembly starting from a suspension of AuNP and a solution of TPyP.

The FTO electrodes were initially cleaned with 3:1 $\mathrm{H}_{2} \mathrm{SO}_{4}: \mathrm{H}_{2} \mathrm{O}_{2}$ solution for $15 \mathrm{~min}$ and washed with deionized water (resistivity $>18 \mathrm{M} \Omega \mathrm{cm}$ ). Then, the electrodes were activated with a 4:1:1 solution of $\mathrm{H}_{2} \mathrm{O}: \mathrm{H}_{2} \mathrm{O}_{2}: \mathrm{NH}_{4} \mathrm{OH}$ for $15 \mathrm{~min}$ and washed again with deionized water.

These electrodes were then modified with a $1.0 \times 10^{-3} \mathrm{~mol} \mathrm{~L}^{-1}$ solution of 3-mercaptopropyltrimethoxysilane (MPTS) for $12 \mathrm{~h}$ in methanol. After modification with MPTS, the electrodes were washed with methanol and used as a substrate for the deposition of the AuNP/TPyP hybrid material.

The FTO-MPTS electrodes were dipped for $10 \mathrm{~min}$

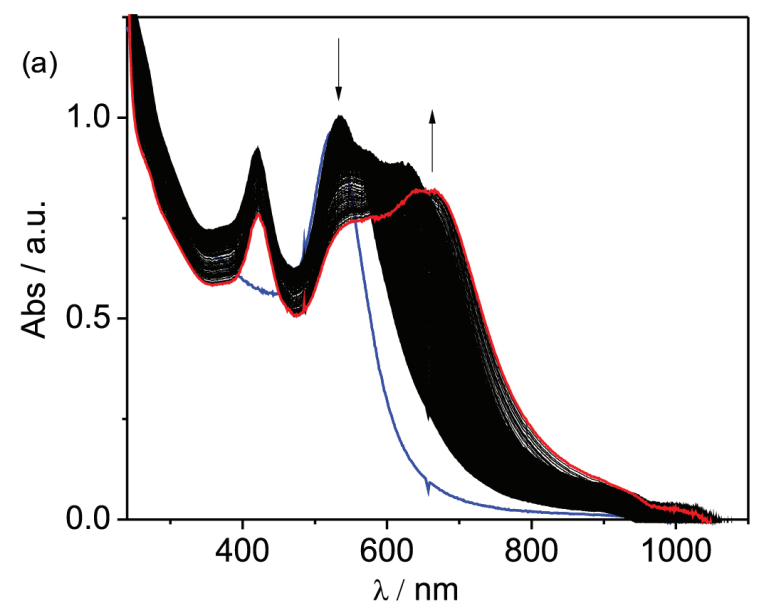

in the AuNP aqueous suspension, then washed with a deionized water/methanol solution (1:1 v:v), then dipped in a $1.0 \times 10^{-4} \mathrm{~mol} \mathrm{~L}^{-1} \mathrm{TPyP}$ solution in dichloromethane for $10 \mathrm{~min}$, and washed again with a mixture of dichloromethane/ methanol (1:1 v:v). This procedure was repeated 10 times in order to assemble the hybrid material.

\section{Results and Discussion}

Probing AuNP-TPyP interaction by UV-Vis spectroscopy and surface-enhanced Raman spectroscopy

AuNPs and TPyP have very different physicochemical properties. AuNPs are hydrophilic and dispersible in water, whereas TPyP is soluble in organic solvents such as chloroform or dichloromethane. Considering such a media compatibility problem, their interaction was evaluated using UV-Vis spectroscopy, as can be seen in Figure 1.

Isolated citrate stabilized AuNPs of about $20 \mathrm{~nm}$ dispersed in water have a typical absorption band with a maximum around $521 \mathrm{~nm}$, assigned to a LSPR band. However, when they approach another particle enough (distance less than about two diameters) they start to interact leading to the appearance of a second band attributed to a plasmonic coupling band, whose position strongly depends on the relative distance of the particles, or the size of the molecular spacer or the bridging ligand. ${ }^{9,22}$ Accordingly, the colloidal destabilization and consequent aggregation can be easily monitored by the intensity of such a plasmonic coupling band.

As can be seen in Figure 1, the addition of TPyP dichloromethane solution leads to nanoparticle aggregation, since the four pyridyl groups of the TPyP have high affinity and coordinate onto AuNPs surface acting as molecular bridges connecting them together.

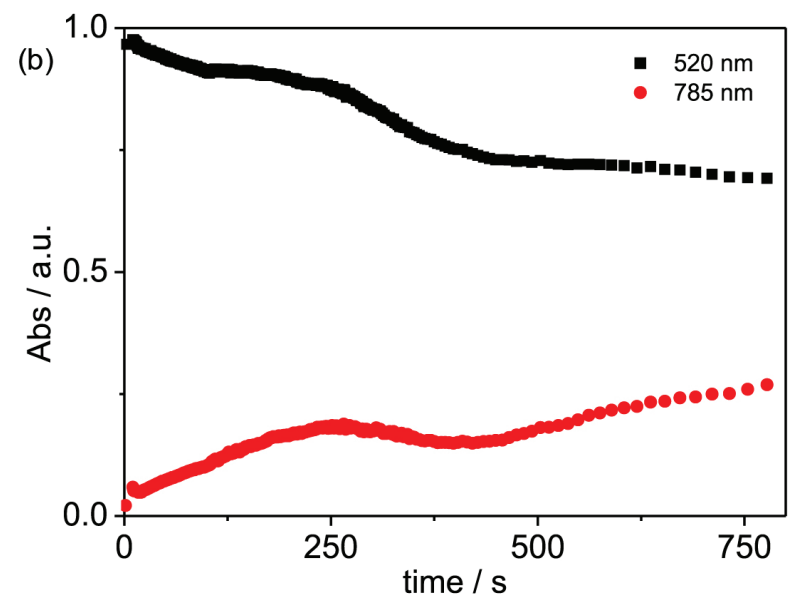

Figure 1. Aggregation process of AuNPs in the aqueous dispersion in the presence of TPyP (a) UV-Vis spectra and (b) kinetics following 520 and 785 nm signals. 
Such a process is likely to occur in two steps: firstly, the binding of TPyP should decrease the dispersibility of the nanoparticles given its highly hydrophobic character and then, secondly, leading to the approaching and binding from one particle to another, thus causing a fast aggregation and subsequent precipitation of the hybrid material.

These two processes control the speed and pattern of aggregation/precipitation of nanoparticles that heavily depend on the concentration of the bridging TPyP molecule and AuNPs, as shown in Figure S2 (Supplementary Information section). Considering an AuNP dispersion with LSPR values at 1.0 unit, no precipitation was observed up to $800 \mathrm{~s}$ after the addition of TPyP concentrations lower than $1.25 \times 10^{-6}\left(5.00 \times 10^{-7}, 2.50 \times 10^{-7}\right.$ and $\left.1.25 \times 10^{-7} \mathrm{~mol} \mathrm{~L}^{-1}\right)$, indicating that the amount of that species is not sufficient to bridge all AuNPs. However, clearly, there is the rise of the plasmonic coupling band at $700 \mathrm{~nm}$ indicating the formation of small aggregates or clusters probably with TPyP molecules surrounded by AuNPs, thus conferring hydrophilic character and relatively high dispersibility to the hybrid material. When that concentration was increased to $1.25 \times 10^{-6} \mathrm{~mol} \mathrm{~L}^{-1}$, a rapid aggregation and precipitation process was observed, indicating that probably this is the optimal concentration for interparticle bridging to take place, but rapid precipitation was also observed with $1 \times 10^{-5}, 5 \times 10^{-6}$ and $2.5 \times 10^{-6} \mathrm{~mol} \mathrm{~L}^{-1}$ of TPyP with the concomitant rise of the plasmon coupling band at $700 \mathrm{~nm}$. Accordingly, the SERS effect generated by nanoparticles was used to study the mode of interaction of the TPyP molecules with the surface of AuNPs, as shown in Figure 2.

Although the vibrational study of the interaction of metallic nanoparticles in aqueous solution with tetrapyridylporphyrins has not been reported yet, the Raman and resonant Raman spectra of porphyrins were extensively studied during the 1970 s to 1990 s. $^{23,24}$ Accordingly, such results were employed to interpret the conformation of TPyP on AuNPs in the hybrid nanomaterials.

TPyP can bind onto the AuNPs surface basically in two ways: (i) through the pyridine groups $\mathrm{N}$-atoms, or (ii) through the porphyrin ring central pyrroles $\mathrm{N}$-atoms, since the free-base macrocycle is being considered here. However, the binding mode can be influenced by surface concentration, where the standing up edge on conformation is more favored at larger concentration, whereas the face-on interaction is more likely at much lower concentrations. In fact, the change in the binding mode is easily observed by analyzing the change in SERS spectra as a function of the TPyP concentration. A sharp and intense peak assigned to $\delta$ (py) appeared around $680 \mathrm{~cm}^{-1}$ as the concentration increased above $2.5 \times 10^{-6} \mathrm{~mol} \mathrm{~L}^{-1}$, whilst at concentrations equal to or less than $5.0 \times 10^{-7} \mathrm{~mol} \mathrm{~L}^{-1}$ the peak around

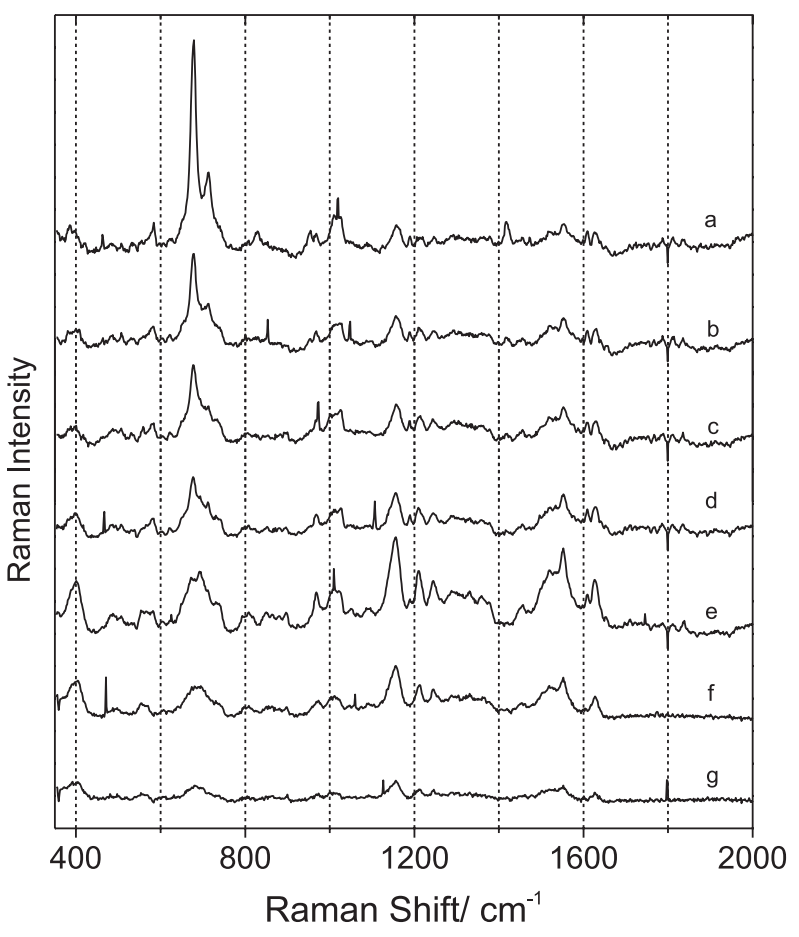

Figure 2. SERS spectra of the hybrid composites generated after interaction of AuNPs dispersion with TPyP and kinetic measurements, using final TPyP concentrations equal to (a) $1.0 \times 10^{-5}$, (b) $5 \times 10^{-6}$, (c) $2.5 \times 10^{-6}$, (d) $1.25 \times 10^{-6}$, (e) $5.0 \times 10^{-7}$, (f) $2.5 \times 10^{-7}$ and (g) $1.25 \times 10^{-7} \mathrm{~mol} \mathrm{~L}^{-1}$.

$1150 \mathrm{~cm}^{-1}$ (assigned to $\delta($ pyr)) was intensified. Such a behavior of the SERS spectra, leading to an increase of $\delta$ (pyr) signals and a decrease in $\delta($ py) signals, and vice versa at lower and higher concentrations of TPyP, can be attributed to a change in the conformation of the molecule on the AuNP surface from face on to edge-on, in accordance with our expectations. The main SERS signals and the respective tentative assignments are listed in Table 1.

\section{Assembling the AuNP/TPyP hybrid on FTO electrodes}

Although TPyP and AuNPs have very different solvation characteristics, they still can interact generating AuNP/TPyP hybrids on the FTO electrode surface. The electrodes were first cleaned and activated as described in the Experimental section and modified with MPTS, thus providing strongly coordinating thiol groups on the electrode surface for the binding of AuNPs by simple immersion in a methanolic solution for $10 \mathrm{~min}$. After this first modification step with AuNPs, the electrodes were carefully washed with methanol (an intermediate solvent that allows the removal of the excess of both AuNP and TPyP), then immersed in the TPyP solution to form the hybrid and washed again with methanol. This simple procedure was repeated ten times while monitoring the 
Table 1. The peak position of the main vibrational modes of TPyP SERS spectra as a function of concentration, and respective tentative assignments

\begin{tabular}{|c|c|c|c|c|c|c|c|c|}
\hline$[\mathrm{TPyP}] /\left(\mathrm{mol} \mathrm{L}^{-1}\right)$ & $1.0 \times 10^{-5}$ & $5.0 \times 10^{-6}$ & $2.5 \times 10^{-6}$ & $1.25 \times 10^{-6}$ & $5.0 \times 10^{-7}$ & $2.5 \times 10^{-7}$ & $1.25 \times 10^{-7}$ & $\begin{array}{c}\text { Tentative } \\
\text { assignment }\end{array}$ \\
\hline & $385(w)$ & $392(w)$ & $392(w)$ & $397(w)$ & $400(\mathrm{~m})$ & $402(\mathrm{~s})$ & $403(\mathrm{~s})$ & $\delta$ porph \\
\hline & $583(w)$ & $580(w)$ & $578(w)$ & $580(w)$ & $560(w)$ & $555(w)$ & $553(w)$ & $\mathrm{A}_{1 \mathrm{~g}}$ \\
\hline & $678(s)$ & $676(s)$ & $678(\mathrm{~s})$ & $677(\mathrm{~s})$ & $691(s)$ & $688(\mathrm{~s})$ & $682(s)$ & $\delta($ py $)$ \\
\hline & & & & $808(w)$ & $808(w)$ & $805(w)$ & & $\mathrm{A}_{1 \mathrm{~g}}$ \\
\hline & $828(w)$ & $830(w)$ & & & $850(w)$ & $863(w)$ & & $\mathrm{B}_{1 \mathrm{~g}}$ or $\mathrm{B}_{2 \mathrm{~g}}$ \\
\hline & $954(w)$ & $968(w)$ & & $968(w)$ & $968(w)$ & $973(w)$ & & $v\left(\mathrm{C}_{\mathrm{m}}\right.$-py $)$ \\
\hline \multirow[t]{7}{*}{ Peak positions $/ \mathrm{cm}^{-1}$} & $1157(w)$ & $1156(\mathrm{~m})$ & $1156(\mathrm{~m})$ & $1156(\mathrm{~s})$ & $1155(\mathrm{~s})$ & $1156(\mathrm{~s})$ & $1156(\mathrm{~s})$ & $\delta(\mathrm{pyr})$ \\
\hline & $1212(w)$ & $1209(w)$ & $1214(w)$ & $1209(w)$ & $1211(w)$ & $1212(\mathrm{~m})$ & $1211(w)$ & $\delta(\mathrm{pyr})$ \\
\hline & $1246(w)$ & $1244(w)$ & $1243(w)$ & $1244(w)$ & $1244(w)$ & $1244(w)$ & $1246(w)$ & $\delta\left(\mathrm{C}_{\mathrm{m}}\right.$-py $)$ \\
\hline & & & $1294(w)$ & $1288(w)$ & $1288(w)$ & $1288(w)$ & & $\delta(\mathrm{py})$ \\
\hline & & & & & $1331(w)$ & $1331(w)$ & & $v\left(\mathrm{C}_{\alpha}-\mathrm{N}\right)$ \\
\hline & & & & & $1457(w)$ & $1454(w)$ & & $v\left(\mathrm{C}_{\alpha}-\mathrm{C}_{\beta}\right)$ \\
\hline & $1554(w)$ & $1552(\mathrm{w})$ & $1552(\mathrm{~m})$ & $1552(\mathrm{~s})$ & $1552(\mathrm{~s})$ & $1552(\mathrm{~s})$ & $1552(w)$ & $\delta($ py) \\
\hline
\end{tabular}

w: weak; m: medium; s: strong; $\delta$ : bending; v: stretching; py: pyridine; pyr: pyrrole; porph: porphyrin.

build-up by UV-Vis spectroscopy, as can be observed in Figure 3 , in order to get a large enough amount of material deposited on the FTO surface.

Analyzing the spectra obtained during the formation of the AuNP/TPyP hybrid, it is interesting to note that the plasmonic band of AuNPs is too low to be observed in the first deposition step and the TPyP Soret band (around $400 \mathrm{~nm}$ ) is more intense. Nevertheless, after the second deposition step of the AuNPs hybrid, the plasmonic coupling band become more intense while the Soret band is broadened and flattened, such that the spectra is dominated almost exclusively by the AuNPs spectral profile after about five deposition steps.

As can be seen in Figure 3b, the deposition of the hybrid nanomaterial on the electrode occurs linearly, indicating

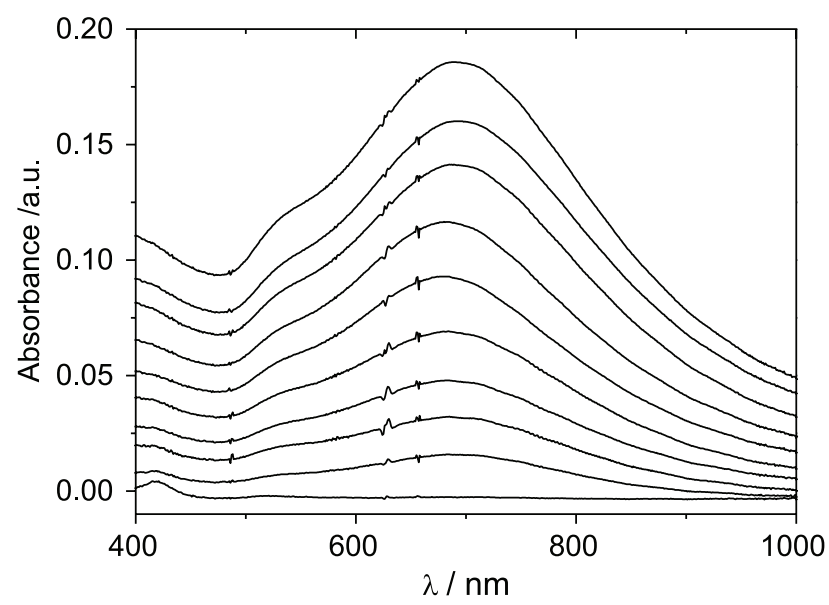

that the same amount of hybrid material is deposited in each dipping cycle in the suspension of AuNPs and TPyP solution, as expected for a reproducible and homogeneous formation/deposition process.

Analyzing the respective UV-Vis spectra obtained, it can be noted that the position of the plasmon coupling band remained almost unchanged during the deposition process indicating that the interparticle distances and chemical environment remained more or less constant, except for a slight bathochromic shift with each deposited step. In fact, the wavelength is very close to that observed when the nanoparticles are aggregated in aqueous suspension. This observation is extremely important, since the measurements in the solution can be used to estimate the time required for the complete interaction of AuNPs with TPyP, and this can

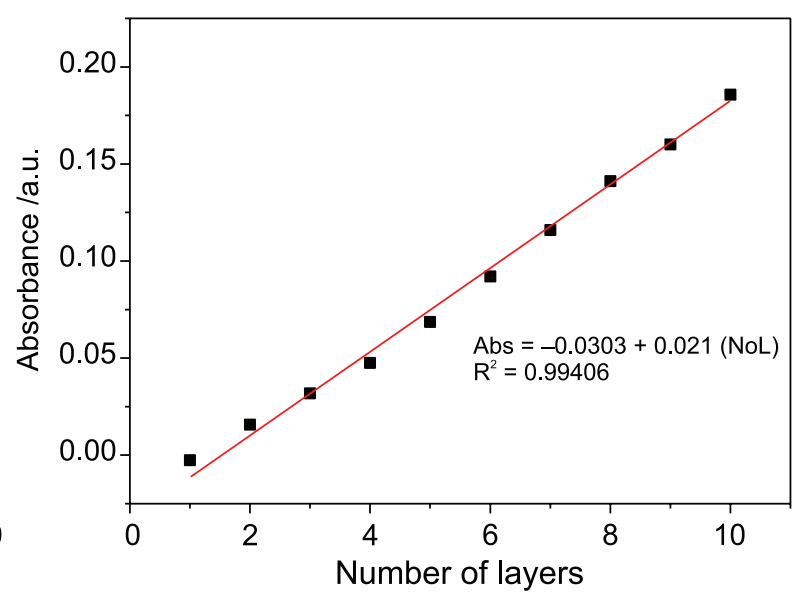

Figure 3. (a) Spectrophotometric monitoring of the stepwise coordinative build-up of AuNP/TPyP hybrid on FTO surface, and (b) plot of absorbance at $700 \mathrm{~nm}$ as a function of a number of deposition steps. 
be used to estimate a suitable time for the deposition steps. A FEG-SEM image of the sample after ten deposition steps is shown in Figure 4.

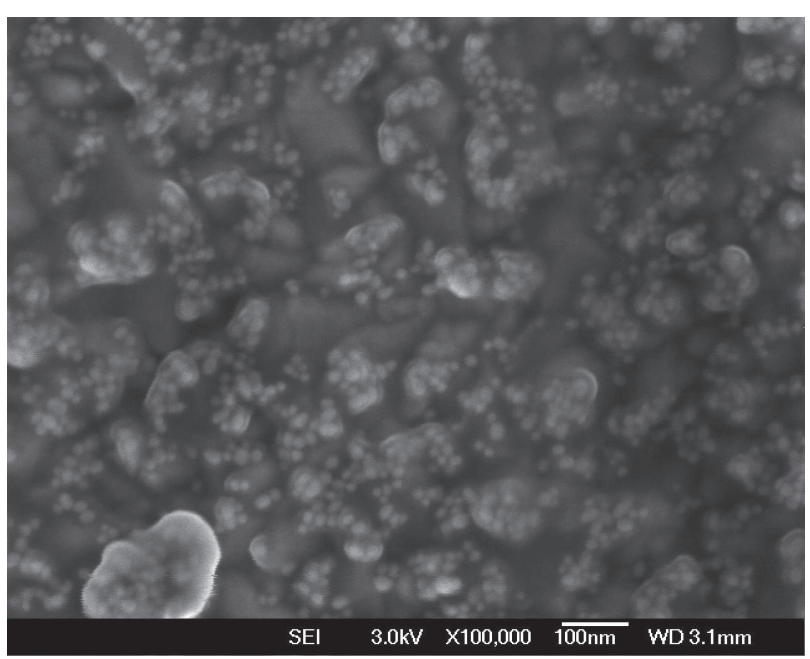

Figure 4. FEG-SEM image of an FTO electrode after ten deposition steps of the AuNP/TPyP hybrid material.

Although TPyP-assisted AuNPs deposition process on the FTO electrode is homogeneous from the point of view of the amount deposited at each step, the particles are not homogeneously distributed along the electrode surface, as can be seen in the FEG-SEM image of Figure 4. In fact, the deposition only can occur on some specific sites, where there is MPTS, but the low density of initial sites did not interfere in the subsequent deposition steps that appear to occur more laterally, rather than according to the traditional models that often suggest a tridimensional growth of the nanoparticle hybrids.

Considering that the main reason for the modification of the FTO electrodes is their application as amperometric sensors, the modified electrodes were evaluated by EIS (Figure 5). EIS is a convenient technique to investigate the kinetics of the electron transfer processes at the electrode interface and, in this case, how the AuNP/TPyP hybrid modifies the properties of the unmodified FTO electrode.

The impedance spectra were modeled using a modified Randles circuit (Table 2) composed by the electrolyte resistance $\left(R_{S}\right)$ in series with a constant phase element (Q), that is in parallel with the charge-transfer resistance in the film $\left(\mathrm{R}_{\mathrm{CT}}\right)$, and a Warburg element $(\mathrm{W})$ accounting for the diffusional resistance. These elements have a great numerical similarity, except for RCT and Q.

The semi-circle (at high to medium frequency), in EIS is associated with an active electron transfer $\left(\mathrm{R}_{\mathrm{CT}}\right)$, and a nearly $45^{\circ}$ line (mid to low frequency) that is related to diffusional control processes at the interface (W). The bare FTO electrode presented a semicircle that corresponding to an $\mathrm{R}_{\mathrm{CT}}$ of about $737 \Omega$, which decreased to $445 \Omega$ after modification, indicating that the AuNP/TPyP hybrid deposited on the surface alters the electron transfer kinetics to the ferri/ferrocyanide pair. This decrease in resistivity is explained by the presence of nanostructured hybrid film that have improved the charge transfer process.

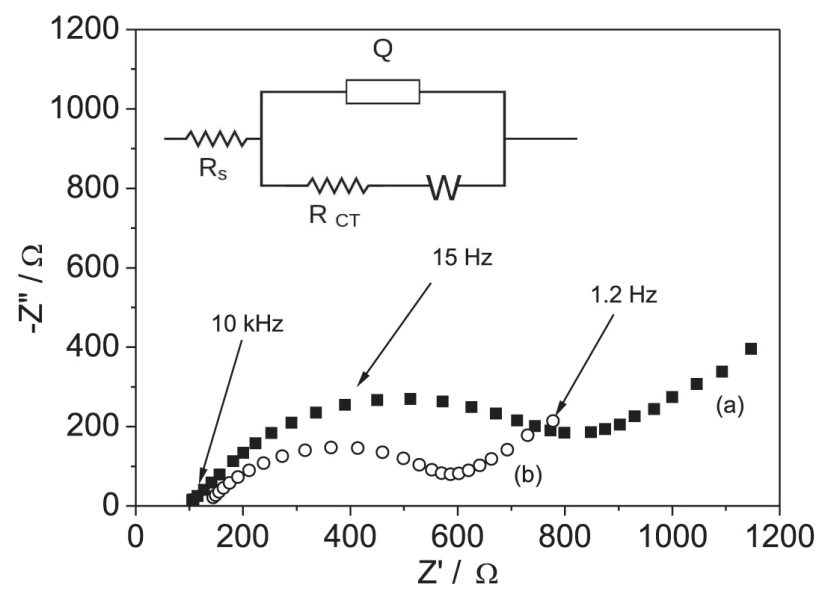

Figure 5. Nyquist plots in $1.0 \times 10^{-3} \mathrm{~mol} \mathrm{~L}^{-1} \mathrm{~K}_{3}\left[\mathrm{Fe}(\mathrm{CN})_{6}\right]: \mathrm{K}_{4}\left[\mathrm{Fe}(\mathrm{CN})_{6}\right]$ (1:1) mixture containing $0.1 \mathrm{~mol} \mathrm{~L}^{-1} \mathrm{KNO}_{3}$ at (a) FTO clean surface and (b) modified with the AuNP/TPyP hybrid. Insert: scheme of the modified Randles type circuit used to model the impedance spectra.

Table 2. EIS data for the $\left[\mathrm{Fe}^{\mathrm{III} / \mathrm{II}}(\mathrm{CN})_{6}\right]^{3-/ 4-}$ redox couple on FTO and FTO-AuNP/TPyP electrodes

\begin{tabular}{lcc}
\hline $\begin{array}{l}\text { Equivalent circuit } \\
\text { elements }\end{array}$ & FTO & FTO AuNp/TPyP \\
\hline $\mathrm{R}_{\mathrm{S}} / \Omega$ & 105 & 145 \\
$\mathrm{R}_{\mathrm{CT}} / \Omega$ & 737 & 445 \\
$\mathrm{Q} / \mathrm{F}$ & $5.1 \times 10^{-6}$ & $1.4 \times 10^{-6}$ \\
$\mathrm{~W} /\left(\Omega \mathrm{s}^{-1 / 2}\right)$ & $5.2 \times 10^{-3}$ & $6.3 \times 10^{-3}$ \\
$\varphi$ & 0.72 & 0.71 \\
\hline
\end{tabular}

$\mathrm{R}_{\mathrm{S}}$ : electrolyte resistance; $\mathrm{R}_{\mathrm{CT}}$ : charge-transfer resistance in the film; $\mathrm{Q}$ : constant phase element; W: Warburg element; $\varphi$ : ideal capacitor coefficient; FTO: fluorine-doped tin oxide; AuNP: gold nanoparticles; TPyP: 5,10,15,20-tetra(4-pyridyl)-21H,23H-porphine.

Oxidation of nitrite and sulfite on AuNP/TPyP hybrid modified FTO electrodes

Nitrite and sulfite are very well known preservatives, respectively, of red meat and juices and wines, but that exhibits poor electrochemical response on FTO electrodes. Thus, the electrocatalytic properties of the modified electrodes were tested for those reducing species envisaging their application for analytic purposes. Measurements were performed in two different $\mathrm{pH}$ conditions to optimize the amperometric responses. Thus, while nitrite detection was performed in $0.01 \mathrm{~mol} \mathrm{~L}^{-1}$ acetate buffer $(\mathrm{pH}=4.70)$, sulfite detection was performed 

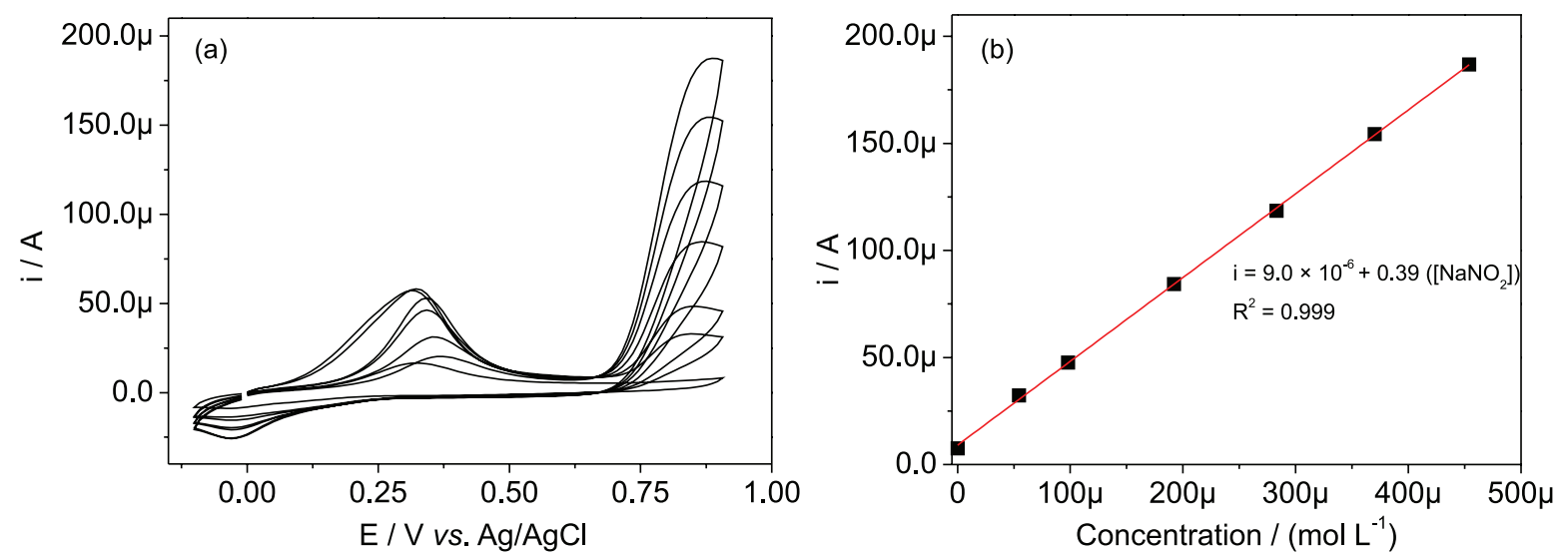

Figure 6. (a) $\mathrm{CVs}_{\mathrm{s}}$ of $\mathrm{NaNO}_{2}$ at AuNP/TPyP hybrid modified FTO electrode, in $0.01 \mathrm{~mol} \mathrm{~L}^{-1}$ acetate buffer $\mathrm{pH}=4.70$ solution, and (b) the plot of i (at $0.8 \mathrm{~V})$ vs. $\left[\mathrm{NaNO}_{2}\right]$.
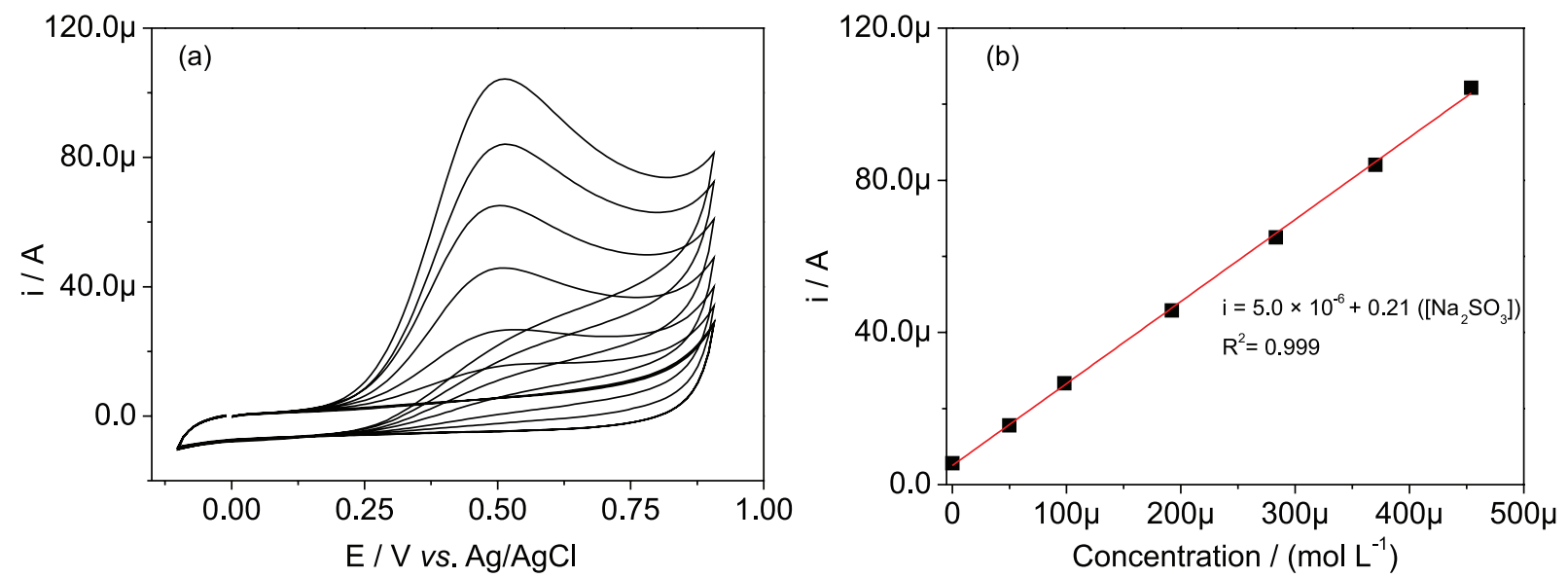

Figure 7. (a) $\mathrm{CVs}$ of $\mathrm{Na}_{2} \mathrm{SO}_{3}$ at AuNP/TPyP hybrid modified FTO electrode, in $0.01 \mathrm{~mol} \mathrm{~L}^{-1}$ phosphate buffer $\mathrm{pH}=6.88$ solution, and (b) the plot of $\mathrm{i}$ (at $0.4 \mathrm{~V}$ ) vs. $\left[\mathrm{Na}_{2} \mathrm{SO}_{3}\right]$.

in $\mathrm{pH}=6.88$ phosphate buffer $\left(0.01 \mathrm{~mol} \mathrm{~L}^{-1}\right)$, and the results shown in Figures 6 and 7.

As already presented in literature, ${ }^{25}$ a broad peak is usually observed for electrocatalytic oxidation reactions of nitrite above $1.2 \mathrm{~V}(v s \mathrm{Ag} / \mathrm{AgCl})$ using unmodified FTO electrodes, that become much sharper in the presence of the modified electrodes (as can be seen in Figure 6a), while the process is shifted to $0.8 \mathrm{~V}$ with an excellent correlation $\left(\mathrm{R}^{2}=0.999\right)$. Interestingly, sulfite was oxidized at $0.5 \mathrm{~V}$ with similarly good linear correlation (Figure 7a) but with about half of the sensitivity (slope of 0.21 as compared to 0.39 ). Consequently, the simultaneous detection of both sulfite and nitrite can be envisaged in pHs larger than about 5 , since both are unstable in strongly acidic medium decomposing, respectively, to $\mathrm{SO}_{2}$ and $\mathrm{H}_{2} \mathrm{O}$, and $\mathrm{NO}, \mathrm{NO}_{3}{ }^{-}$and water.

Electrodes modified with gold nanoparticles already have been used for detection of nitrite or sulfite, ${ }^{26}$ but this is the first report using self-assembled AuNP/TPyP hybrid material whose excellent linear response in the 50 to $500 \mu \mathrm{mol} \mathrm{L}{ }^{-1}$ is noteworthy. For example, the hybrid material prepared using trinuclear ruthenium clusters ${ }^{9}$ and AuNPs also exhibited enhanced electrocatalytic properties for nitrite oxidation but the sensitivity of the porphyrinbased material is much higher $\left(39.0 \times 10^{-2} \mu \mathrm{A} \mathrm{L} \mu \mathrm{mol}^{-1}\right.$ as compared to $12.8 \times 10^{-2} \mu \mathrm{A} \mathrm{L} \mu \mathrm{mol}^{-1}$ ), thus lowering the limit of detection. This significant increase in sensitivity can be directly related to the TPyP bridging ligands since it is an aromatic macrocyclic ring slightly smaller than the ruthenium clusters that favor the electronic interactions of the gold nanoparticles.

\section{Conclusions}

Even considering the quite different polar characteristics, water-dispersed AuNPs were shown to interact with 5,10,15,20-tetra(4-pyridyl)-21 $H, 23 H$-porphine (TPyP) forming a hybrid nanomaterial. The kinetics of such a process was monitored by UV-Vis spectroscopy showing that it is strongly influenced by TPyP concentration, such that aggregation/precipitation was not observed at relatively 
low concentrations (less than $1.25 \times 10^{-6} \mathrm{~mol} \mathrm{~L}^{-1}$ ), but small clusters that remain in suspension were rather formed. It has demonstrated by SERS that the binding conformation of TPyP on AuNPs surface changes from a face on to edge-on as the surface concentration increase, leading to the enhancement of the pyridine-related SERS signals and decrease of porphyrin ring signals. The deposition of small amounts of AuNP/TPyP hybrid increased the conductivity of the FTO electrode ( $815 \Omega$ (FTO) to $512 \Omega$ (AuNP/TPyP modified FTO)), as confirmed by electrochemical impedance spectroscopy measurements, but increased much more significantly the electrocatalytic properties for the oxidation of nitrite and sulfite. In fact, FTO electrodes modified with AuNP/TPyP presented an electrocatalytic activity much higher than the corresponding cluster derivative and well behave response and sensitivity for both species, as demonstrated by the excellent linear behavior for quantification in the 50 to $500 \mu \mathrm{mol} \mathrm{L}^{-1}$ range.

\section{Supplementary Information}

Supplementary information (containing electronic spectrum, size distribution histogram, UV-Vis spectra monitoring the kinetics of the aggregation process of AuNPs) is available free of charge at http://jbcs.sbq.org.br as PDF file.

\section{Acknowledgments}

J. J. Santos thanks Conselho Nacional de Desenvolvimento Científico e Tecnológico for the scholarship (CNPq 140418/2008-3). S. H. Toma thanks CNPq for the Scholarship in Technological Development Productivity (CNPq 305950/2016-9). K. Araki thanks FAPESP (18/21489-1), Petróleo Brasileiro (Petrobras 0050.0101557.16.9), and CNPq (401581/2016-0 and 303137/2016-9) for financial support. This study was financed in part by the Coordenação de Aperfeiçoamento de Pessoal de Nível Superior - Brasil (CAPES, Finance Code 001).

\section{References}

1. Reguera, J.; Langer, J.; de Aberasturi, D. J.; Liz-Marzán, L. M.; Chem. Soc. Rev. 2017, 46, 3866.

2. Toma, H. E.; Zamarion, V. M.; Toma, S. H.; Araki, K.; J. Braz. Chem. Soc. 2010, 21, 1158.

3. Toma, S. H.; Santos, J. J.; Araki, K.; Toma, H. E.; Anal. Chim. Acta 2015, 855, 70.
4. Santos, J. J.; Toma, S. H.; Corio, P.; Araki, K.; J. Raman Spectrosc. 2017, 48, 1190.

5. Bentley, C. L.; Kang, M.; Unwin, P. R.; J. Am. Chem. Soc. 2019 , $141,2179$.

6. Chiang, H.-C.; Wang, Y.; Zhang, Q.; Levon, K.; Biosensors 2019, 9, 50.

7. Zhao, Y.; Wu, Y.; Zhang, Y.; Chen, Z.; Cao, X.; Di, J.; Yang, J.; Electroanalysis 2009, 21, 939.

8. Daniel, M.-C.; Astruc, D.; Chem. Rev. 2004, 104, 293.

9. Toma, S. H.; Santos, J. J.; Araki, K.; Toma, H. E.; Eur J. Inorg. Chem. 2011, 1640.

10. Fagadar-Cosma, E.; Sebarchievici, I.; Lascu, A.; Creanga, I.; Palade, A.; Birdeanu, M.; Taranu, B.; Fagadar-Cosma, G.; J. Alloys Compd. 2016, 686, 896.

11. Zhang, Q.; Fan, G.; Chen, W.; Liu, Q.; Zhang, X.; Zhang, X.; Liu, Q.; Biosens. Bioelectron. 2020, 150, 111846.

12. Zhang, W.; Lai, W.; Cao, R.; Chem. Rev. 2017, 117, 3717.

13. Araki, K.; Angnes, L.; Toma, H. E.; Adv. Mater. 1995, 7, 554.

14. Nogueira, A. F.; Furtado, L. F. O.; Formiga, A. L. B.; Nakamura, M.; Araki, K.; Toma, H. E.; Inorg. Chem. 2004, 43, 396.

15. Mayer, I.; Eberlin, M. N.; Tomazela, D. M.; Toma, H. E.; Araki, K.; J. Braz. Chem. Soc. 2005, 16, 418.

16. Martins, P. R.; Popolim, W. D.; Nagato, L. A. F.; Takemoto, E.; Araki, K.; Toma, H. E.; Angnes, L.; Penteado, M. D. C.; Food Chem. 2011, 127, 249.

17. Yang, H.; Yin, D.; Gao, L.; Zhang, X.; Zhang, X.; Liu, Q.; J. Colloid Interface Sci. 2020, 561, 881.

18. Vecchi, A.; Erickson, N. R.; Sabin, J. R.; Floris, B.; Conte, V.; Venanzi, M.; Galloni, P.; Nemykin, V. N.; Chem.-Eur. J. 2015, $21,269$.

19. Toma, S. H.; Santos, J. J.; Velho, R. G.; Nakamura, M.; Toma, H. E.; Araki, K.; Electrochim. Acta 2012, 66, 287.

20. Turkevich, J.; Stevenson, P. C.; Hillier, J.; Discuss. Faraday Soc. 1951, 11, 55.

21. Frens, G.; Nature (London), Phys. Sci. 1973, 241, 20.

22. Chegel, V.; Rachkov, O.; Lopatynskyi, A.; Ishihara, S.; Yanchuk, I.; Nemoto, Y.; Hill, J. P.; Ariga, K.; J. Phys. Chem. C 2012, 116, 2683.

23. Araki, K.; Santos, P. S.; Toma, H. E.; Spectrosc. Lett. 1993, 26, 1417.

24. Spiro, T. G.; Burke, J. M.; J. Am. Chem. Soc. 1976, 98, 5482.

25. Rahman, M. M.; Li, X. B.; Lopa, N. S.; Lee, J. J.; Bull. Korean Chem. Soc. 2014, 35, 2072.

26. Maduraiveeran, G.; Ramaraj, R.; J. Anal. Sci. Technol. 2017, $8,14$.

Submitted: January 13, 2020

Published online: April 29, 2020 